\title{
On Numerical Calculation of Transonic Flow Patterns*
}

\author{
By S. Bergman, J. G. Herriot and T. G. Kurtz
}

1. Introduction. Obtaining a mathematical expression for the solution of a problem is frequently not the chief difficulty in developing a practical numerical procedure. This is often the case when the expression involves infinite processes such as integration and differentiation. It then becomes necessary to approximate these infinite processes by some finite process which can be carried out with the use of a computer.

The purpose of the present paper is to describe a numerical procedure for computing families of transonic flow patterns for compressible fluids. The basis for this procedure is provided by a family of particular solutions of the Chaplygin equation (2.1). These solutions were obtained independently by Bergman [1], [2] and by Bers and Gelbart [6], [7].

Although these solutions have a fairly simple mathematical form, they involve multiple integrals of complicated functions which must be approximated in some way in order to make their numerical computation feasible. This difficulty is overcome by approximating the functions involved by polynomials and then integrating these polynomials.

When applying "the method of particular solutions" to solve boundary-value problems, we form a linear combination

$$
\Psi^{(N)}=\sum_{\nu=1}^{N} A_{\nu} \psi_{\nu}
$$

of particular solutions $\psi_{\nu}$ and determine the coefficients $A_{\nu}$ so that $\Psi^{(N)}$ approximates the prescribed boundary values. Thus, the computation of a set of particular solutions is of interest not only in itself but also as a first step in the solution of certain boundary-value problems.

2. The Partial Differential Equations Arising in the Theory of Compressible Fluid Flow. It is well known that the partial differential equation satisfied by the stream function $\psi$ of a two-dimensional, inviscid, irrotational, compressible fluid flow when considered in the physical plane (i.e., when $\psi$ is considered as a function of the coordinates $x, y$ of the plane in which the flow takes place) is nonlinear. However, if we consider the equation in the hodograph plane, i.e., if we consider $\psi$ as a function of the speed $v$ and the angle $\theta$ which the velocity vector makes with a fixed direction (say, with the $x$-axis), then, as Chaplygin [8] and Molenbroek [11] have shown, $\psi$ satisfies the linear partial differential equation

$$
\frac{1-M^{2}}{\rho^{2}} \frac{\partial^{2} \psi}{\partial \theta^{2}}+\frac{v}{\rho}-\frac{\partial}{\partial v}\left(\frac{v}{\rho} \frac{\partial \psi}{\partial v}\right)=0 .
$$

Received August 4, 1967.

* NSF GP 5962 and AF 1047-66. 
Here $\rho$ denotes the density which is related to the pressure $p$ by the relation $p=c \rho^{k}$, where $c$ and $k$ are constants ( $k=1.4$ for air). The Mach number $M$ and the density $\rho$ are related to the speed $v$ by

$$
\begin{aligned}
\rho & =\left(1-(k-1) v^{2} / 2\right)^{1 /(k-1)}, \\
M & =\frac{v}{\left(1-(k-1) v^{2} / 2\right)^{1 / 2}} .
\end{aligned}
$$

Since the denominator in (2.3) is the local speed of sound, $M$ will be smaller than one if the flow is subsonic and larger than one if it is supersonic. Instead of the speed $v$ we may introduce the variable

$$
H=\int_{v_{1}}^{v} \frac{\rho}{v} d v=\int_{v_{1}}^{v}\left(1-\frac{k-1}{2} v^{2}\right)^{1 /(k-1)} \frac{d v}{v},
$$

where $v_{1}$ denotes the velocity corresponding to $M=1$. If one introduces the potential $\phi$ as well as the stream function $\psi$, then the differential equations connecting these quantities assume the form

$$
\frac{\partial \phi}{\partial \theta}=\frac{\partial \psi}{\partial H}, \quad \frac{\partial \phi}{\partial H}=-l(H) \frac{\partial \psi}{\partial \theta}, \quad l(H)=\frac{1-M^{2}}{\rho^{2}} .
$$

(See [3], [10].)

From (2.5) we deduce at once that the differential equation for $\psi$ may be written in the form

$$
\frac{\partial^{2} \psi}{\partial H^{2}}+l(H) \frac{\partial^{2} \psi}{\partial \theta^{2}}=0 .
$$

Equation (2.6) is a differential equation of mixed type, since $l(H)>0$ for $H<0$ (i.e., for $v<v_{1}, M<1$ ), while $l(H)<0$ for $H>0$ (i.e., for $v>v_{1}, M>1$ ).

In order to transform a solution of $(2.6)$ from the $(H, \theta)$-plane to the physical plane, we use the relations

$$
\begin{aligned}
& x=\int\left[\frac{\left(M^{2}-1\right) \cos \theta}{\rho v^{2}} \psi_{\theta}-\frac{\sin \theta}{\rho v} \psi_{v}\right] d v+\left[\frac{\cos \theta}{\rho} \psi_{v}-\frac{\sin \theta}{\rho v} \psi_{\theta}\right] d \theta \\
& y=\int\left[\frac{\left(M^{2}-1\right) \sin \theta}{\rho v^{2}} \psi_{\theta}+\frac{\cos \theta}{\rho v} \psi_{v}\right] d v+\left[\frac{\sin \theta}{\rho} \psi_{v}+\frac{\cos \theta}{\rho v} \psi_{\theta}\right] d \theta .
\end{aligned}
$$

If the integration is along a streamline, $\psi_{v} d v+\psi_{\theta} d \theta=0$ and (2.7) becomes

$$
\begin{aligned}
& x=\int \frac{\cos \theta}{\rho}\left[\frac{M^{2}-1}{v^{2}} \psi_{\theta} d v+\psi_{v} d \theta\right], \\
& y=\int \frac{\sin \theta}{\rho}\left[\frac{M^{2}-1}{v^{2}} \psi_{\theta} d v+\psi_{v} d \theta\right] .
\end{aligned}
$$

Note that if the Jacobian of this transformation vanishes at a point, the transformation can fail to be one-to-one in a neighborhood of the point, and the flow may have no physical significance in the neighborhood. See, e.g., $[5$, p. 17] or $[10$, p. $311 \mathrm{ff}$.$] .$ 
3. The Particular Solutions and Their Approximation. The particular solutions of (2.5) and (2.6), with which we are concerned, are a generalization of the harmonic polynomials obtained by taking real and imaginary parts of the analytic functions $(\theta+i H)^{n}$. These solutions $\phi_{n, j}(H, \theta)$ and $\psi_{n, j}(H, \theta), j=1,2$, $n=0,1, \cdots$, may be written in the form

$$
\begin{aligned}
\phi_{n, 1}(H, \theta)+i \psi_{n, 1}(H, 9) & =(\theta+i H)^{[n]} \\
\equiv & {\left[\theta^{n}-2 !\left(\begin{array}{c}
n \\
2
\end{array}\right) \theta^{n-2} p_{2}(H)+4 !\left(\begin{array}{c}
n \\
4
\end{array}\right) \theta^{n-4} p_{4}(H)+\cdots\right] } \\
& +i\left[1 !\left(\begin{array}{c}
n \\
1
\end{array}\right) \theta^{n-1} s_{1}(H)-3 !\left(\begin{array}{c}
n \\
3
\end{array}\right) \theta^{n-3} s_{3}(H)+\cdots\right],
\end{aligned}
$$

$$
\begin{aligned}
\phi_{n, 2}(H, \theta)+i \psi_{n, 2}(H, \theta) & =i \odot(\theta+i H)^{[n]} \\
\equiv & -\left[1 !\left(\begin{array}{c}
n \\
1
\end{array}\right) \theta^{n-1} p_{1}(H)-3 !\left(\begin{array}{c}
n \\
3
\end{array}\right) \theta^{n-3} p_{3}(H)+\cdots\right] \\
& +i\left[\theta^{n}-2 !\left(\begin{array}{c}
n \\
2
\end{array}\right) \theta^{n-2} s_{2}(H)+4 !\left(\begin{array}{c}
n \\
4
\end{array}\right) \theta^{n-4} s_{4}(H)-\cdots\right]
\end{aligned}
$$

where

$$
\begin{aligned}
& p_{0}(H)=1, \quad p_{1}(H)=\int_{H_{0}}^{H} l\left(H_{1}\right) d H_{1} \\
& p_{m}(H)=\int_{H_{0}}^{H} l\left(H_{m}\right) \int_{H_{0}}^{H_{m}} p_{m-2}\left(H_{m-1}\right) d H_{m-1} d H_{m}
\end{aligned}
$$

and

$$
\begin{aligned}
& s_{m}(H)=\int_{H_{0}}^{H} \int_{H_{0}}^{H_{m}} l\left(H_{m-1}\right) s_{m-2}\left(H_{m-1}\right) d H_{m-1} d H_{m}, \\
& s_{0}(H)=1, \quad s_{1}(H)=H-H_{0}=\int_{H_{0}}^{H} d H_{1} .
\end{aligned}
$$

(See [2], [3] and [6].) It will be convenient to choose $H_{0}=0$ corresponding to $M=1$.

Because of the nature of the function $l(H)$, the integrals involved in the calculation of $s_{m}(H)$ would be very difficult to evaluate exactly. Therefore, we approximate $l(H)$ in suitable intervals by polynomials and then integrate these polynomials to obtain polynomial approximations for the functions $s_{m}(H)$. The intervals are

$$
J_{1}:-1.0 \leqq H \leqq 0 \quad \text { corresponding to } .284 \leqq M \leqq 1,
$$

and

$$
J_{2}: 0 \leqq H \leqq 0.2 \text { corresponding to } 1 \leqq M \leqq 1.78 \text {. }
$$

The polynomial approximation of $l(H)$ in $J_{\kappa}$ is denoted by

$$
\tilde{l}_{\kappa}(H)=\sum_{\nu=0}^{10} A_{\nu}{ }^{(\kappa)} \eta_{\kappa}{ }^{\nu}, \quad \kappa=1,2, \quad \eta_{1}=H, \quad \eta_{2}=5 H .
$$


In order to determine the coefficients of the approximating polynomial in these intervals, we need an algorithm for the calculation of $l(H)$ as a function of $H$. We consider the special case of air, for which $k=1.4$. Since $\rho$ and $M$ are functions of $v$, as shown by Eqs. (2.2) and (2.3), $l(H)$ can be expressed as a function of $v$ using its definition in (2.5). In this special case the integral in (2.4) can be evaluated in closed form and yields

$$
\begin{array}{r}
H=\left\{\left(1-0.2 v^{2}\right)^{1 / 2}\left[\frac{\left(1-0.2 v^{2}\right)^{2}}{5}+\frac{1-0.2 v^{2}}{3}+1\right]\right. \\
\left.-\frac{1}{2} \ln \frac{1+\left(1-0.2 v^{2}\right)^{1 / 2}}{1-\left(1-0.2 v^{2}\right)^{1 / 2}}\right\}\left.\right|_{v_{1}} ^{v}
\end{array}
$$

where $v_{1}=(5 / 6)^{1 / 2}$. We use Newton's method to calculate $v$ corresponding to any value of $H$ in $J_{1}$ or $J_{2}$ and then $l(H)$ can be calculated at once.

The Remez algorithm [12], as adapted for the Burroughs B5500 computer by Golub and Smith [9], was used to determine polynomials $l_{\kappa}(H)$ which are the best approximations to $l(H)$ in the Chebyshev sense on the intervals $J_{k}$. The values of the coefficients $A_{\nu}{ }^{(\kappa)}, \kappa=1,2, \nu=0,1, \cdots, 10$, are given in Table 1 . From the approximation algorithm we know that

\section{TABLE 1}

Values of the coefficients $A_{\nu}{ }^{(\alpha)}$ of the approximations to $l(H)$, see (3.5)

\begin{tabular}{r|r|r}
\hline${ }^{\kappa}$ & \multicolumn{1}{|c}{} & \multicolumn{1}{c}{2} \\
\hline 0 & 0.0001711222 & -0.0025985374 \\
1 & -9.3681945493 & -1.3189899758 \\
2 & -55.284916968 & -22.598654078 \\
3 & -248.40627057 & 280.04321217 \\
4 & -840.40978809 & -2002.2845890 \\
5 & -2052.0104328 & 8142.5278038 \\
6 & -3488.0712341 & -20236.235647 \\
7 & -3982.8542755 & 31196.920595 \\
8 & -2896.2958023 & -29181.568535 \\
9 & -1207.8174204 & 15188.597028 \\
10 & -219.39926509 & -3388.9118171 \\
\hline
\end{tabular}

$$
\begin{aligned}
& \max _{J_{1}}\left|l(H)-\tilde{l}_{1}(H)\right| \leqq 1.7 \times 10^{-4} \\
& \max _{J_{2}}\left|l(H)-\tilde{l}_{2}(H)\right| \leqq 2.6 \times 10^{-3} .
\end{aligned}
$$

The function $l(H)$ becomes very large and negative when $H$ exceeds 0.2 . Indeed, as $H \rightarrow 0.2513, M \rightarrow \infty, v \rightarrow \sqrt{ } 5$ and $l(H) \rightarrow-\infty$. It was therefore practically impossible to obtain a good polynomial approximation for $H>0.2$.

The polynomial approximations for the $s_{m}(H)$ in the interval $J_{\kappa}$, denoted by $\tilde{s}_{m}{ }^{(\kappa)}(H)$, satisfy the recurrence relation given by (3.4) and can be expressed as 


$$
\begin{aligned}
& \tilde{s}_{2}{ }^{(\kappa)}(H)=H^{2} \sum_{\nu=0}^{10} C_{\nu}{ }^{(2, \kappa)} \eta_{\kappa}{ }^{\nu}, \quad C_{\nu}{ }^{(2, \kappa)}=\frac{A_{\nu}{ }^{(\kappa)}}{(\nu+1)(\nu+2)}, \quad \kappa=1,2, \\
& \tilde{s}_{3}{ }^{(\kappa)}(H)=H^{3} \sum_{\nu=0}^{10} C_{\nu}{ }^{(3, \kappa)} \eta_{\kappa}{ }^{\nu}, \quad C_{\nu}{ }^{(3, \kappa)}=\frac{A_{\nu}{ }^{(\kappa)}}{(\nu+2)(\nu+3)}, \quad \kappa=1,2,
\end{aligned}
$$

and, in general,

$$
\tilde{s}_{m}{ }^{(\kappa)}(H)=H^{m} \sum_{\nu=0}^{10[m / 2]} C_{\nu}^{(m, \kappa)} \eta_{\kappa}{ }^{\nu} \quad \kappa=1,2,
$$

where

$$
C_{\nu}^{(m, \kappa)}=\sum_{\{\xi \mid 0 \leqq \xi \leq 10,0 \leqq \nu-\xi \leqq 10[(m-2) / 2]\}} \frac{A_{\xi}^{(\kappa)} C_{\nu-\xi}^{(m-2, \kappa)}}{(\nu+m-1)(\nu+m)} .
$$

(Note that $[m / 2]=m / 2$ if $m$ is even but [m/2] $=(m-1) / 2$ if $m$ is odd.) Letting $\tilde{s}_{m}(H)=\tilde{s}_{m}{ }^{(k)}(H)$ for $H$ in $J_{k}$, the approximations to the stream functions $\psi_{n, 1}$ and $\psi_{n, 2}$, denoted by $\tilde{\psi}_{n, 1}$ and $\tilde{\psi}_{n, 2}$, are calculated using Eqs. (3.1) and (3.2) with $s_{m}(H)$ replaced by $\tilde{s}_{m}(H)$. It is clear that the approximations satisfy

$$
\tilde{\psi}_{H H}+\tilde{l}(H) \tilde{\psi}_{\theta \theta}=0, \quad \tilde{l}(H)=\tilde{l}_{\kappa}(H) \text { for } H \text { in } J_{\kappa} .
$$

4. Computational Results. It is seen from Table 1 that the coefficients of the polynomials $\tilde{l}_{k}(H)$ are very large; they are of constant sign in $\tilde{l}_{1}(H)$, which is used for $H<0$, and of alternating sign in $\tilde{l}_{2}(H)$, which is used for $H>0$. Hence, in both cases the evaluation of these functions and of the functions ${\overrightarrow{S_{m}}}^{(\kappa)}(H)$ will involve large terms of alternating sign and this may result in the loss of several significant digits. The magnitude of the error given in (3.7) indicates that single-precision arithmetic (about 11 decimal digits) on the Stanford Burroughs B5500 computer is adequate for the calculation of $\tilde{l}_{k}(H)$. However, for the evaluation of $\tilde{s}_{m}{ }^{(\kappa)}(H)$ and the subsequent calculation of $\tilde{\psi}_{n, j}$, single-precision arithmetic was found to be inadequate. Hence these calculations were carried out in double-precision arithmetic (78-bit arithmetic equivalent to about 22 decimal digits) on the B5500. Some typical values of $\tilde{\psi}_{n, j}$ are given in Table 2 .

As a measure of how well the approximate solutions satisfy the differential equation (2.6), we introduce the quantity

$$
M_{G}(\tilde{\psi})=\left[\frac{\iint_{G}|S(\tilde{\psi})|^{2} d H d \theta}{\iint_{G}|\tilde{\psi}|^{2} d H d \theta}\right]^{1 / 2}
$$

where

$$
S(\tilde{\psi})=\partial^{2} \Psi / \partial H^{2}+l(H) \partial^{2} \tilde{\psi} / \partial \theta^{2} .
$$

Since $\tilde{\psi}_{n, j}$ satisfies (3.12), we see that

$$
S\left(\tilde{\psi}_{n, j}\right)=[l(H)-\tilde{l}(H)] \partial^{2} \tilde{\psi}_{n, j} / \partial \theta^{2} .
$$

By formally differentiating Eqs. (3.1) and (3.2), formulas are obtained for $\partial^{2} \tilde{\psi}_{n, j} / \partial \theta^{2}$ and these are used to evaluate $S\left(\tilde{\psi}_{n, j}\right)$. 


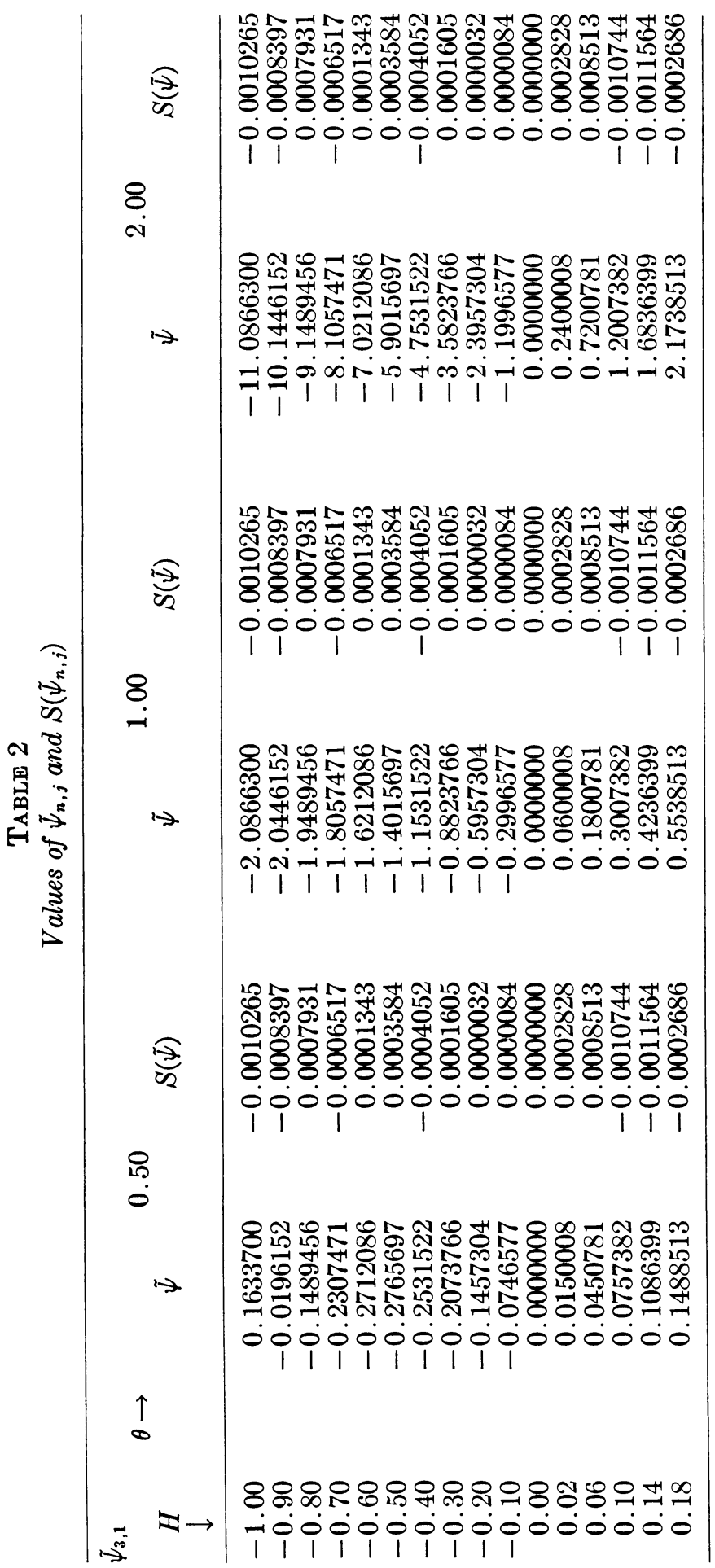




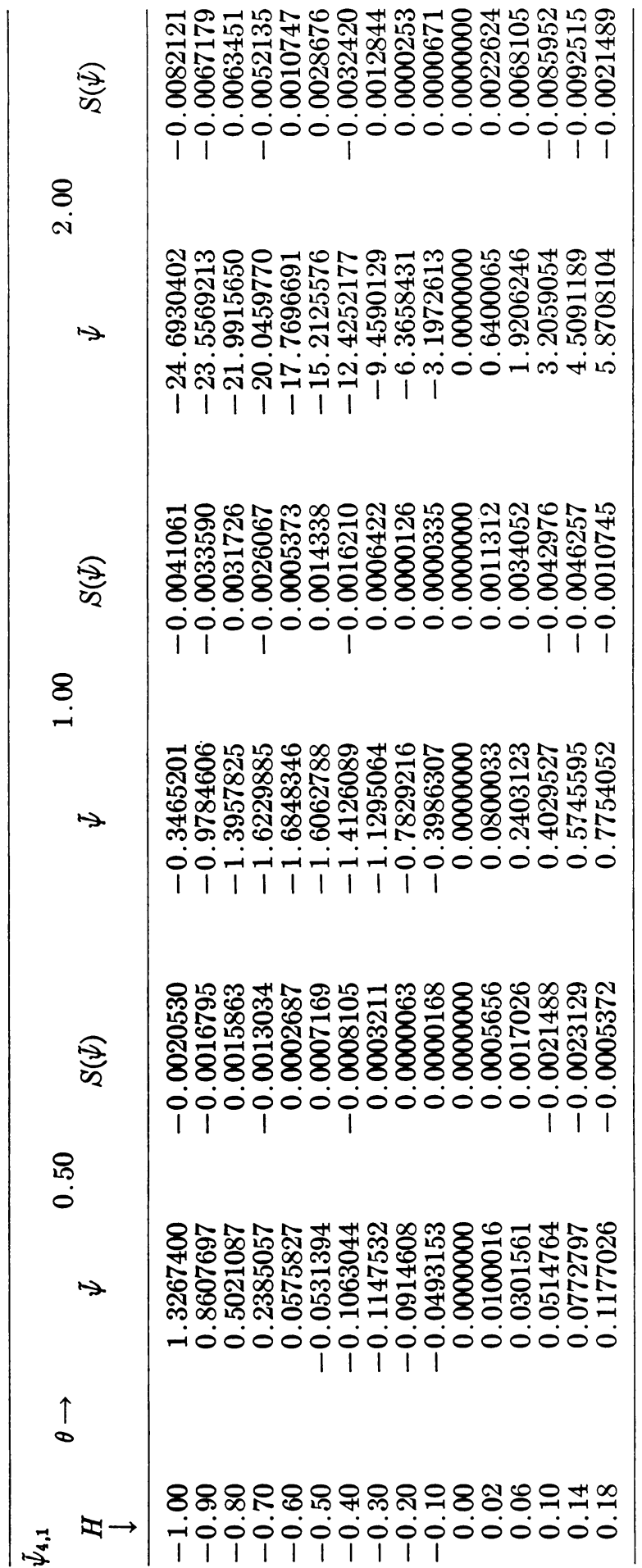




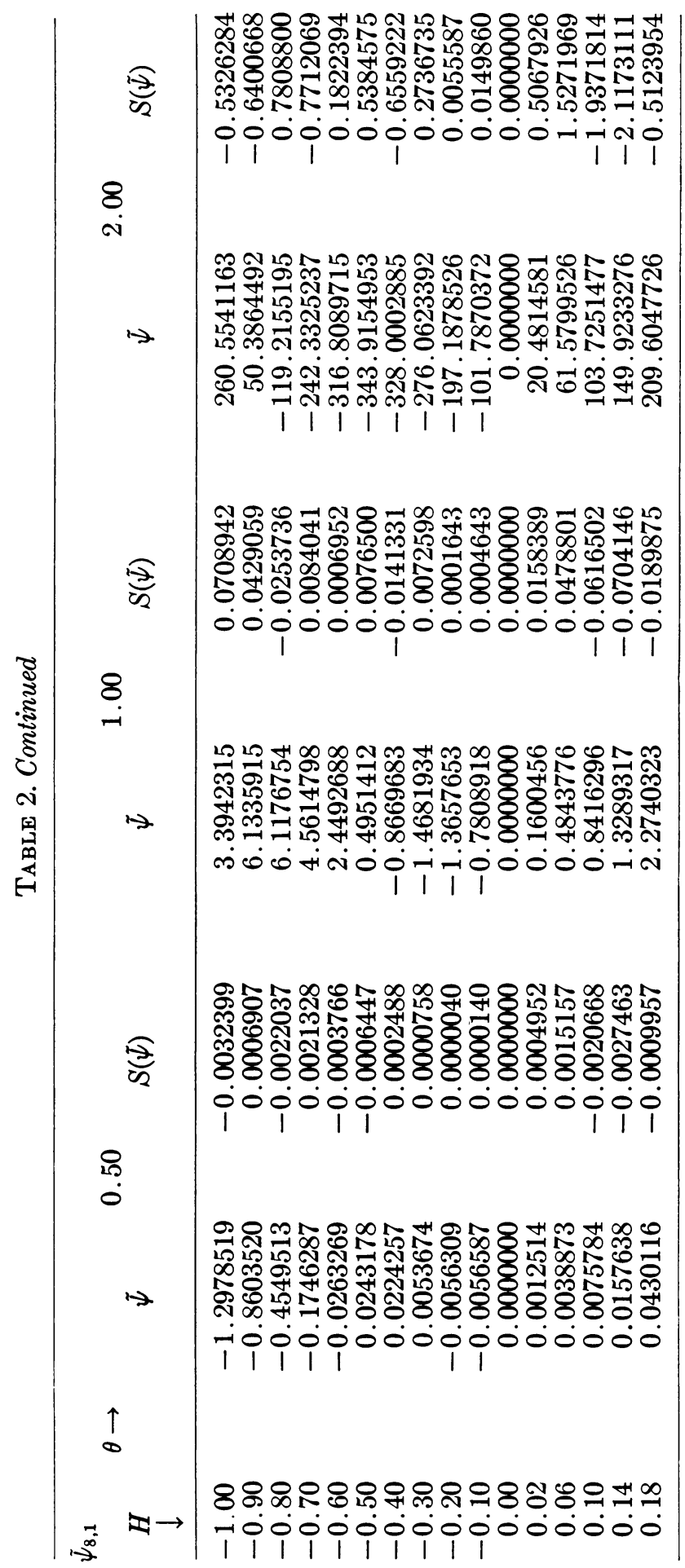




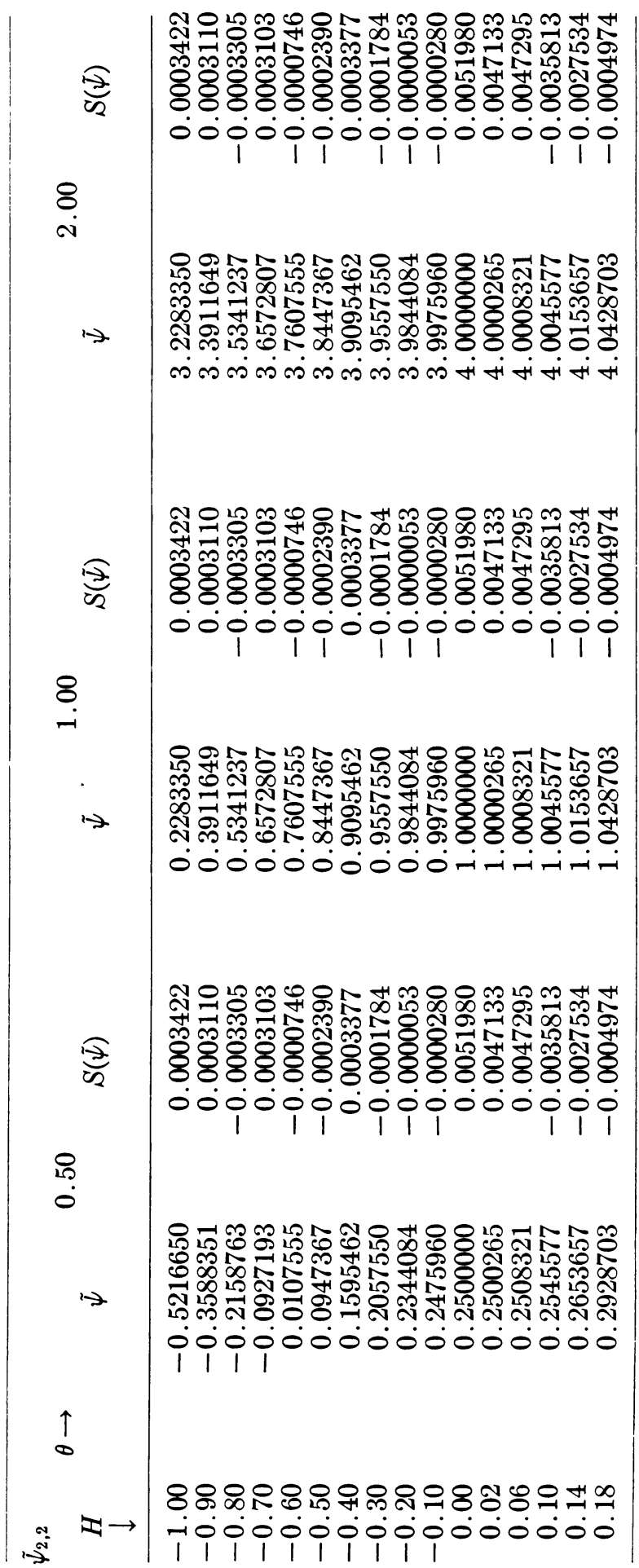




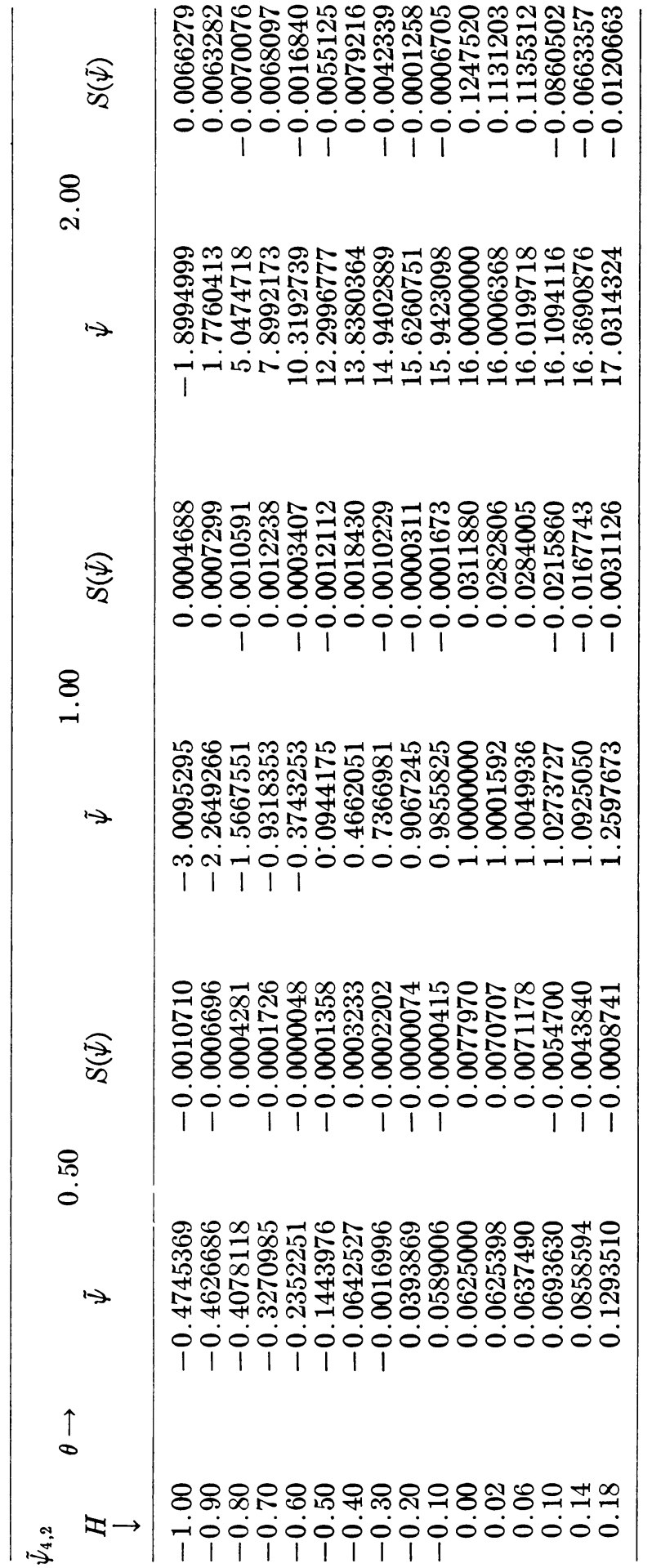




\begin{tabular}{|c|c|}
\hline$\underset{\mathscr{V}}{\mathscr{S}}$ & 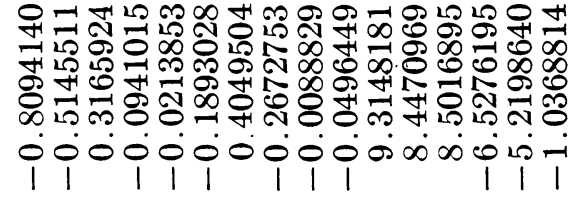 \\
\hline$\underset{i}{8}$ & \\
\hline$\rightarrow$ & 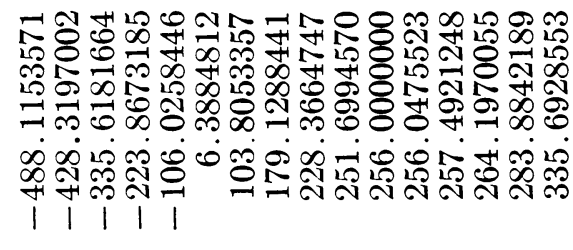 \\
\hline$\stackrel{\mathbb{Z}}{\mathscr{V}}$ & 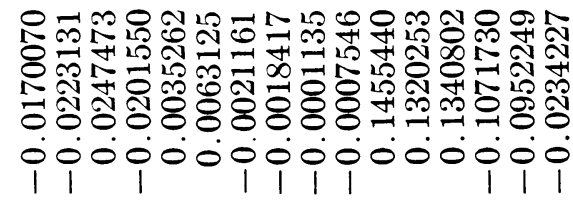 \\
\hline 8 & \\
\hline$\rightarrow$ & 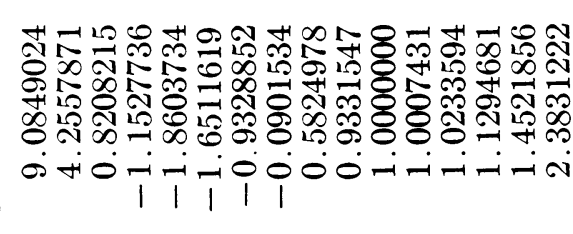 \\
\hline$\sqrt{\frac{3}{2}}$ & 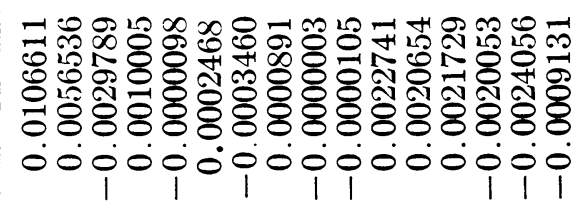 \\
\hline $\begin{array}{l}\text { : } \\
0\end{array}$ & \\
\hline$\rightarrow$ & 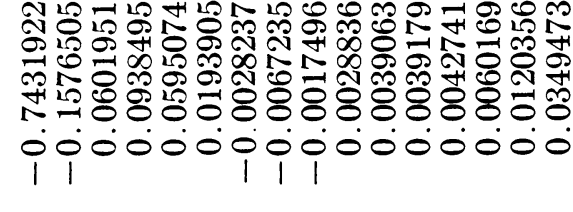 \\
\hline $\begin{array}{l}\uparrow \\
0\end{array}$ & \\
\hline$\nabla \rightarrow$ & 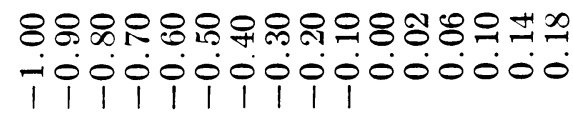 \\
\hline
\end{tabular}


These calculations were also carried out in double-precision arithmetic on the B5500. Typical values for $S\left(\tilde{\psi}_{n, j}\right)$ are given in Table 2 and for $G=$ $\{H, \theta \mid-1.0 \leqq H \leqq .2,0 \leqq \theta \leqq 2\}$ we have

$$
M_{G}\left(\tilde{\psi}_{n, 1}\right) \leqq 0.01 \quad \text { when } n \leqq 7,
$$

and

$$
M_{G}\left(\tilde{\psi}_{n, 2}\right) \leqq 0.01 \quad \text { when } n \leqq 5
$$

Streamlines for three particular examples,

$$
\begin{aligned}
& \tilde{\psi}^{(1)}=5 \tilde{\psi}_{1,1}+\tilde{\psi}_{5,1}+\tilde{\psi}_{4,2}, \\
& \tilde{\psi}^{(2)}=5 \tilde{\psi}_{1,1}+\tilde{\psi}_{4,1}+\tilde{\psi}_{4,2},
\end{aligned}
$$

and

$$
\tilde{\psi}^{(3)}=5 \tilde{\psi}_{1,1}+\tilde{\psi}_{3,1}+\tilde{\psi}_{3,2},
$$
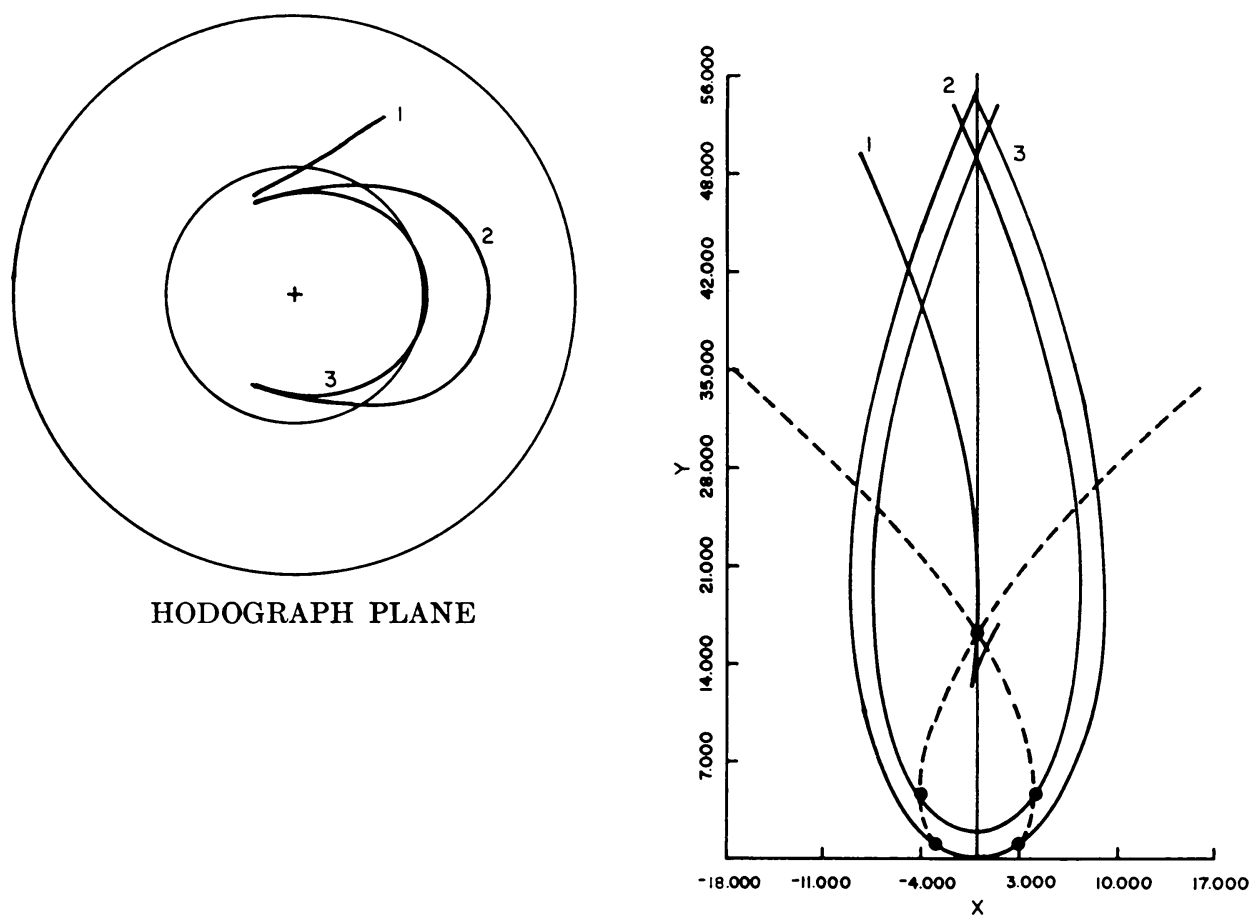

PHYSICAL PLANE

FIGURE 1. $\tilde{\psi}^{(1)}=5 \tilde{\psi}_{1,1}+\tilde{\psi}_{5,1}+\tilde{\psi}_{4,2}$

Streamline $1 \quad \tilde{\psi}^{(1)}=\mathbf{5 . 0 6 2 5}$

Streamline $2 \quad \tilde{\psi}^{(1)}=1.0000$

Streamline $3 \quad \psi^{(1)}=\mathbf{0 . 0 6 2 5}$

_... - Sonic Line

- Transition Points 
were computed in the hodograph plane and transformed into the physical plane by the numerical evaluation of the integrals in (2.7) using the trapezoidal rule. The results of these computations are indicated in Figs. 1-3.

As noted above, the transformation from the $(H, \theta)$-plane to the physical plane may fail to be one-to-one. Consequently, the flows represented by the above streamfunctions correspond to physically possible flows only in certain subdomains. This explains why some of the streamlines drawn intersect. It should also be observed that not all of the intersections of the streamlines with the sonic line (shown as a dotted line) correspond to transitions between subsonic and supersonic flow. Those intersections which do correspond to such transitions are indicated on the figures.

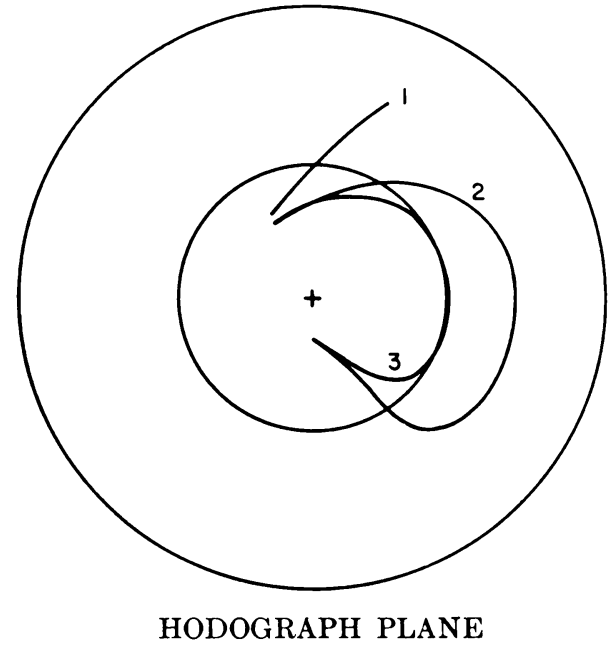

HODOGRAPH PLANE

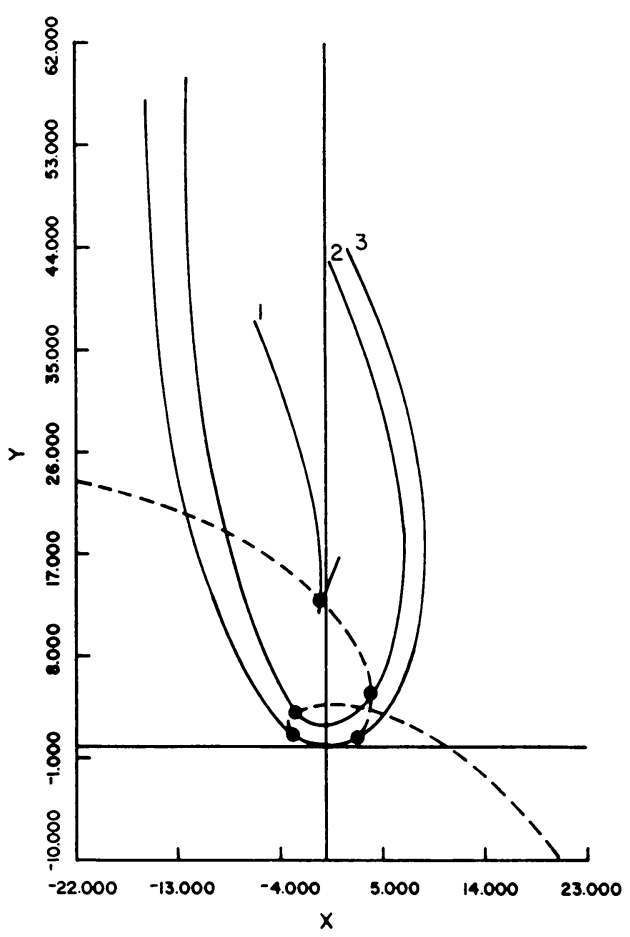

PHYSICAL PLANE

FIGURE 2. $\tilde{\psi}^{(2)}=5 \tilde{\psi}_{1,1}+\tilde{\psi}_{4,1}+\tilde{\psi}_{4,2}$

Streamline $1 \quad \tilde{\psi}^{(2)}=\mathbf{5 . 0 6 2 5}$

Streamline $2 \quad \tilde{\psi}(2)=1.0000$

Streamline $3 \quad \tilde{\psi}^{(2)}=0.0625$

- . - - Sonic Line

- Transition points

Remark. Stark [13], [14] has used a set of particular solutions generated by integral operators as a basis for another numerical procedure for computing solutions of (2.1). More detailed numerical results from the present procedure can be found in [4]. 


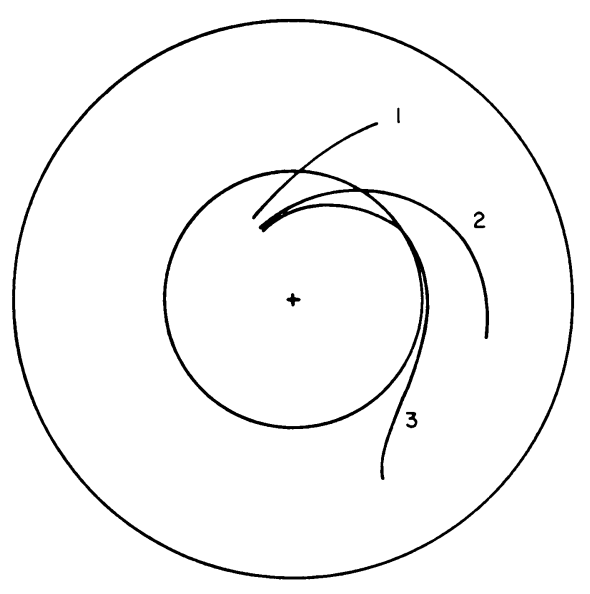

HODOGRAPH PLANE

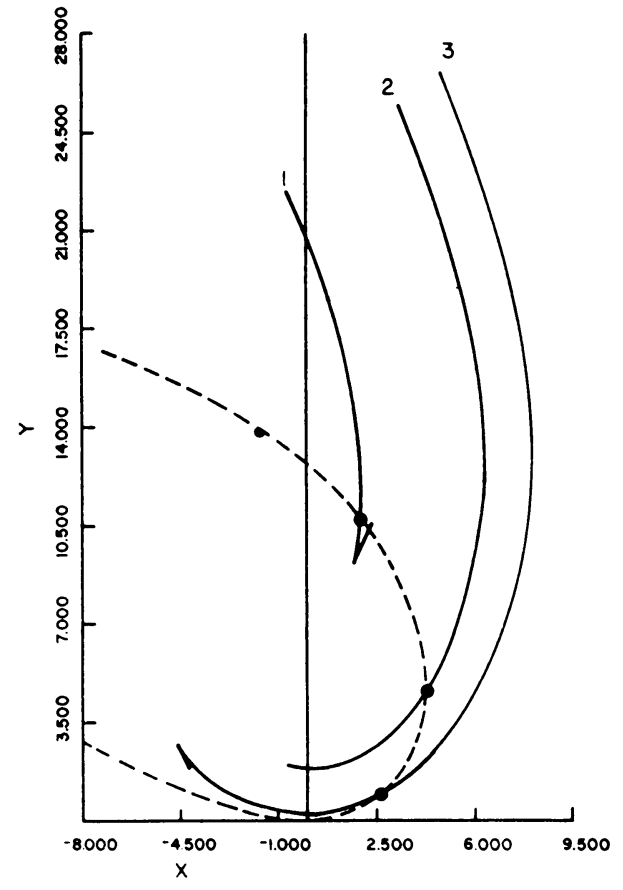

PHYSICAL PLANE

FIGURE 3. $\tilde{\psi}^{(3)}=5 \tilde{\psi}_{1,1}+\tilde{\psi}_{3,1}+\tilde{\psi}_{3,2}$

Streamline $1 \quad \tilde{\psi}^{(3)}=3.375$

Streamline $2 \tilde{\psi}^{(3)}=1.000$

Streamline $3 \quad \tilde{\psi}^{(8)}=0.125$

---- Sonic Line

- Transition Points

Department of Mathematics

Department of Computer Sciences

Stanford University

Stanford, California

1. S. Bergman, "The hodograph method in the theory of compressible fluids," Supplement to Fluid Dynamics by R. von Mises and K. O. Friedrichs, Brown University, Providence, R. I., 1942.

2. S. Bergman, "A formula for the stream function of certain flows," Proc. Nat. Acad. Sci. U.S.A., v. 29, 1943, pp. 276-281. MR 5, 247.

3. S. BERGMAN, "On representation of stream functions of subsonic and supersonic flows of compressible fluids," J. Rational Mech. Anal., v. 4, 1955, pp. 883-905. MR 17, 549.

4. S. Bergman, J. G. Herriot \& T. G. KURTz, Numerical Calculation of Transonic Flow Patterns, Tech. Rep. No. CS 51, Computer Science Department, Stanford University, 1966.

5. L. Bers, Mathematical Aspects of Subsonic and Transonic Gas Dynamics, Surveys in Appl. Math., Vol. 3, Wiley, New York, 1958. MR $20 \# 2960$.

6. L. BERS \& A. GELBART, "On a class of differential equations in the mechanics of continua," Quart. Appl. Math., v. 1, 1943, pp. 168-188. MR 5, 25.

7. L. Bers \& A. Gelbart, "On a class of functions defined by partial differential equations," Trans. Amer. Math. Soc., v. 56, 1944, pp. 67-93. MR 6, 86.

8. S. A. Chaplygin, On Gas Jets, Scientific Memoirs, Moscow University MathematicsPhysics Section, 21, 1904, pp. 1-121; English transl., published by NACA TM 1063, 1044. MR 7, 495 . 
9. G. H. Golub \& L. B. Sмiтh, Chebyshev Approximation of Continuous Functions by a Chebyshev's System of Functions, Tech. Rep. No. CS 72, Computer Science Dept., Stanford University, 1967.

10. R. von Mises, Mathematical Theory of Compressible Fluid Flow, Applied Math. and Mech., v. 3, Academic Press, New York, 1958. MR 20 \#1504.

11. P. Molenbroek, "Über einige Bewegungen eines Gases mit Annahme eines Geschwindigkeitspotentials," Arch. Math. Phys. (2), v. 9, 1890, pp. 157-195.

12. E. YA. Remez, General Computational Methods of Cebyšev Approximation. The Problems with Linear Real Parameters, Izdat. Akad. Nauk Ukrain. SSR, Kiev, 1957; English transl., Books 1 and 2, AEC-tr-4491, U. S. Atomic Energy Commission. MR 19, 580.

13. J. M. STARK, "Application of Bergman's integral operators to transonic flows," Internat. J. Non-linear Mech., v. 1, 1966, pp. 17-34.

14. J. M. STARk, Transonic Flow Patterns Generated by Bergman's Integral Operator, Report, Department of Mathematics, Stanford University, 1964. 